\title{
Derivation of the rotation matrix in general rectilinear systems by means of vector and matrix formalism
}

\author{
KAZIMIERz STróż at Institute of Physics and Chemistry of Metals, University of Silesia, Bankowa 12, 40-007 Katowice, Poland. \\ E-mail: kstroz@usctouxl.cto.us.edu.pl
}

(Received 8 March 1996; accepted 17 June 1996)

\begin{abstract}
A generalized form of a symmetry operator has been derived by vector and matrix formalism in contrast to the tensor or dyadic notation most often used in the literature. The final equation is based on relations between real and reciprocal space familiar to crystallographers. Typical applications of the formula are also provided.
\end{abstract}

\section{Introduction}

Space groups as special groups of isometries are subgroups of the Euclidean group (the group of all affine mappings that preserve distances). Each isometric mapping can be obtained by superposition of translation, rotation and inversion. In its representation denoted as $(\mathbf{R}, \mathbf{t})$, the linear or matrix part $\mathbf{R}$ is responsible for the space rotation of rotoinversion. A transformation restricted to a linear part leaves the origin invariant. In a three-dimensional rectilinear system $\mathbf{a}_{1}, \mathbf{a}_{2}, \mathbf{a}_{3}$, it is given by

$$
\left(\begin{array}{l}
x_{1}^{\prime} \\
x_{2}^{\prime} \\
x_{3}^{\prime}
\end{array}\right)=\left(\begin{array}{lll}
R_{11} & R_{12} & R_{13} \\
R_{21} & R_{22} & R_{23} \\
R_{31} & R_{32} & R_{33}
\end{array}\right)\left(\begin{array}{c}
x_{1} \\
x_{2} \\
x_{3}
\end{array}\right),
$$

where $x_{i}$ and $x_{1}^{\prime}$ denote coordinates of a point before and after transformation, respectively. The matrix $\mathbf{R}$ can be equivalently described in geometric terms as a triplet $\mathrm{l}, \alpha, i$ (where the vector $I$ defines the axis of rotation and its sense, $\alpha$ is the angle of rotation and $i$ holds the information about presence of inversion).

All rotation matrices needed for a conventional description of space groups together with the orientation of corresponding symmetry elements are summarized in Tables 11.2 and 11.3 in International Tables for Crystallography (1983). However, sometimes symmetry manipulation must be carried out with nonconventional cells (Le Page, 1987). Other problems like coexistence of symmetry elements with the lattice or error analysis in experimental symmetry-related data will be simplified if the matrix $R$ is calculated in any rectilinear system. For computational purposes, the coordinate systems would be rather characterized by the metric tensor

$$
\mathbf{G}=\left[\begin{array}{lll}
\left(\mathbf{a}_{1} \cdot \mathbf{a}_{1}\right) & \left(\mathbf{a}_{1} \cdot \mathbf{a}_{2}\right) & \left(\mathbf{a}_{1} \cdot \mathbf{a}_{3}\right) \\
\left(\mathbf{a}_{2} \cdot \mathbf{a}_{1}\right) & \left(\mathbf{a}_{2} \cdot \mathbf{a}_{2}\right) & \left(\mathbf{a}_{2} \cdot \mathbf{a}_{3}\right) \\
\left(\mathbf{a}_{3} \cdot \mathbf{a}_{1}\right) & \left(\mathbf{a}_{3} \cdot \mathbf{a}_{2}\right) & \left(\mathbf{a}_{3} \cdot \mathbf{a}_{3}\right)
\end{array}\right]
$$

than by unit-cell parameters $a_{1}, a_{2}, a_{3}, \alpha, \beta$ and $\gamma$.

A generalized form of a symmetry operator is derived and expressed mainly in a tensor notation (Sands, 1982; SchultzPiszachich, 1979) or a dyadic notation [for references to Zachariasen and Patterson, see Sands (1982)]. The explicit expression in the matrix formalism is often restricted to the case of orthogonal bases (e.g. Giacovazzo, 1992) and is not very convenient for computer implementation. In the work presented, an attempt has been made to derive the matrix $\mathbf{R}$ as a function of $\mathbf{I}, \alpha$ and the metric matrix $\mathbf{G}$ by vector calculations and familiar relations between real and reciprocal space without loss of generality.

\section{Derivation}

Let a coordinate system be described by the metric matrix $\mathbf{G}$. In this system, a point $\mathbf{x}$ is mapped to the point $\mathbf{x}^{\prime}$ by the anticlockwise rotation through the angle $x$ about the vector $\mathbf{I}$ (see Fig. 1). A right-handed orthonormal coordinate system $\hat{\mathbf{e}}_{1}, \hat{\mathbf{e}}_{2}, \hat{\mathbf{e}}_{3}$ intrinsic to that transformation is chosen (SchultzPiszachich, 1979):

$$
\begin{aligned}
& \hat{\mathbf{e}}_{1}=\mathbf{e}=\mathbf{l} /|\mathbf{I}|, \\
& \hat{\mathbf{e}}_{2}=\mathbf{r} /|\mathbf{r}|, \\
& \hat{\mathbf{e}}_{3}=\hat{\mathbf{e}}_{1} \times \hat{\mathbf{e}}_{2} .
\end{aligned}
$$

With respect to the new basis, the vector $\mathbf{x}^{\prime}$ can be decomposed as

$$
\mathbf{x}^{\prime}=\left(\mathbf{x} \cdot \hat{\mathbf{e}}_{1}\right) \hat{\mathbf{e}}_{1}+|\mathbf{r}| \cos \alpha \hat{\mathbf{e}}_{2}+|\mathbf{r}| \sin \alpha \hat{\mathbf{e}}_{3},
$$

from which, after the reduction of the vector $\mathbf{r}$,

$$
\mathbf{x}^{\prime}=(1-\cos \alpha)(\mathbf{e} \cdot \mathbf{x}) \mathbf{e}+\cos \alpha \mathbf{x}+\sin \alpha(\mathbf{e} \times \mathbf{x}),
$$

where the symbol e replaces $\hat{\mathbf{e}}_{1}$.

Vector equation (3) is independent of coordinate system and in order to put it into the form of (1) the vectors must be replaced by their coordinate matrices in the $\mathbf{a}_{1}, \mathbf{a}_{2}, \mathbf{a}_{3}$ system. From the relations between the real and inverse space, the scalar

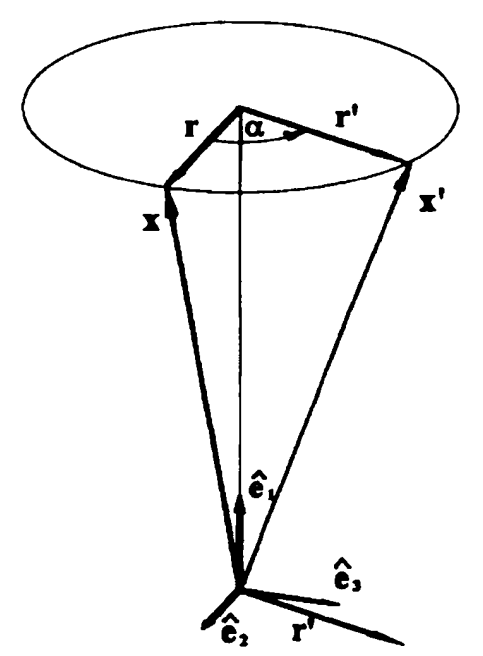

Fig. 1. The orthonormal coordinate system for a rotation matrix derivation. 
product $(\mathbf{e} \cdot \mathbf{x})$ is

$$
\begin{aligned}
(\mathbf{e} \cdot \mathbf{x}) & =\left(\begin{array}{lll}
e_{1} & e_{2} & e_{3}
\end{array}\right) \mathbf{G}\left(\begin{array}{l}
x_{1} \\
x_{2} \\
x_{3}
\end{array}\right) \\
& =\left(\begin{array}{lll}
e_{1}^{*} & e_{2}^{*} & e_{3}^{*}
\end{array}\right)\left(\begin{array}{l}
x_{1} \\
x_{2} \\
x_{3}
\end{array}\right)
\end{aligned}
$$

where $e_{i}$ and $e_{i}^{*}$ denote the coordinates of the unit vector $\mathbf{e}$ in the real $\mathbf{a}_{1}, \mathbf{a}_{2}, \mathbf{a}_{3}$ and the reciprocal $\mathbf{a}_{1}^{*}, \mathbf{a}_{2}^{*}, \mathbf{a}_{3}^{*}$ systems, respectively.

A vector product based on vectors expressed in real space is described in reciprocal space:

$$
\begin{aligned}
(\mathbf{e} \times \mathbf{x})= & \left(\mathbf{a}_{1} \times \mathbf{a}_{2} \cdot \mathbf{a}_{3}\right)\left|\begin{array}{ccc}
\mathbf{a}_{1}^{*} & \mathbf{a}_{2}^{*} & \mathbf{a}_{3}^{*} \\
e_{1} & e_{2} & e_{3} \\
x_{1} & x_{2} & x_{3}
\end{array}\right| \\
= & \left(\begin{array}{c}
\mathbf{a}_{1}^{*} \\
\mathbf{a}_{2}^{*} \\
\mathbf{a}_{3}^{*}
\end{array}\right)[\operatorname{det}(\mathbf{G})]^{1 / 2} \\
& \times\left(e_{2} x_{3}-e_{3} x_{2} e_{3} x_{1}-e_{1} x_{3} \quad e_{1} x_{2}-e_{2} x_{1}\right)
\end{aligned}
$$

and must be recalculated to real space

$$
(\mathbf{e} \times \mathbf{x})=\left(\begin{array}{lll}
\mathbf{a}_{1} & \mathbf{a}_{2} & \mathbf{a}_{3}
\end{array}\right) \operatorname{vol} \mathbf{G}^{*}\left(\begin{array}{l}
e_{2} x_{3}-e_{3} x_{2} \\
e_{3} x_{1}-e_{1} x_{3} \\
e_{1} x_{2}-e_{2} x_{1}
\end{array}\right),
$$

where $\operatorname{vol}=[\operatorname{det}(\mathbf{G})]^{1 / 2}$ is the volume of the unit cell and $\mathbf{G}^{*}=\mathbf{G}^{-1}$ is the metric matrix in reciprocal space.

Replacement of vectors in (3) by their coordinate matrices with the help of (4) and (5) leads to the form (1). An explicit expression for elements of the rotation matrix in the general rectilinear system may be written as

$$
\begin{aligned}
R_{i j}= & e_{i} e_{j}^{*}(1-\cos \alpha)+\delta_{i j} \cos \alpha \\
& +\operatorname{vol}\left(G_{i(j+1)}^{*} e_{(j+2)}-G_{i(j+2)}^{*} e_{(j+1)}\right) \sin \alpha,
\end{aligned}
$$

where the indices in parentheses must be decremented by 3 if their value is greater than 3 and the Kronecker delta $\delta_{i j}=1$ if $i=j$ and 0 if $i \neq j$. The above equation is equivalent to the tensor form of the rotation matrix described by Sands (1982).

\section{Application}

The formula (6) plays an essential role in the matrix presentation of symmetry operations described in conventional or nonconventional crystal cells and in the derivation of some properties of such presentations.

(i) The symmetry axis is parallel to the direction $[u v w]$. Here $I=u \mathbf{a}_{1}+v \mathbf{a}_{2}+w \mathbf{a}_{3}$ and $\alpha=360 / n$, where $n$ is the order of the symmetry axis.

(ii) The symmetry axis is perpendicular to the plane $(h k l)$. The vector $\mathbf{l}^{*}=h \mathbf{a}_{1}^{*}+k \mathbf{a}_{2}^{*}+l \mathbf{a}_{3}^{*}$ is described in reciprocal space and its coordinates must be transformed to real space by the equation $(h k l) \mathbf{G}^{*}=[u v w]$.

Thus, all matrices contained in the previously mentioned tables in International Tables can be obtained and the algorithm for derivation of so-called 'coordinate triplets' from the geometric description of symmetry operations can be extended to nonconventional crystal systems.

(iii) The symmetry axis is parallel to a reciprocal base vector $\mathbf{a}_{i}^{*}$. The matrix $\mathbf{G}^{*}$ holds the components of the reciprocal base vectors referred to real space, so the factors beside $\sin \alpha$ in (6) can be expressed as components of the vector products $\mathbf{a}_{i}^{*} \times \mathbf{e}$, i.e. $R_{i j}=e_{i} e_{j}^{*}(1-\cos \alpha)+\delta_{i j} \cos \alpha+\left(\mathbf{a}_{i}^{*} \times \mathbf{e}\right)_{j} \sin \alpha$. This form is convenient to express the obvious property that the $i$ th coordinate of vector $\mathbf{x}$ will be invariant if the rotation axis is parallel to the reciprocal vector $\mathbf{a}_{i}^{*}$. For example, if the vector $\mathbf{l}$ is parallel to the base vector $\mathbf{a}_{1}^{*}$, then $e_{1}^{*}=1 / e_{1} \neq 0, e_{2}^{*}=e_{3}^{*}=0$ and $\left(\mathbf{a}_{1}^{*} \times \mathbf{e}\right)=0$. Thus, $R_{11}=1, R_{12}=R_{13}=0$ and the coordinate $x_{1}$ of the vector $\mathbf{x}$ will be unchanged on the transformation.

(iv) The trace of the rotation matrix. From (6) it is easy to show that $\operatorname{Tr}(\mathbf{R})$, defined as the sum of diagonal elements $R_{11}+R_{22}+R_{33}$ is equal to $1+2 \cos \alpha$ and is independent of the coordinate system.

\section{References}

Giacovazzo, C. (1992). Fundamentals of Crystallography, edited by C. Giacovazzo, pp. 69-71. IUCr/Oxford University Press.

International Tables for Crystallography (1983). Vol. A. Dordrecht: Reidel.

Le Page, Y. (1987). J. Appl. Cryst. 20, 264-269.

Sands, D. E. (1982). Vectors and Tensors in Crystallography, pp. 101-103. Reading, MA: Addison-Wesley.

Schultz-Piczachich, W. (1979). Tensor algebra und -analysis, pp. 42-44. Leipzig: BSB B.G. Tubner Verlagsgesellschaft. (In German.) 